\title{
Characterization of the thrombin generation profile in systemic lupus erythematosus
}

\author{
A Kern ${ }^{1,2}$, E Barabás ${ }^{1}$, A Balog ${ }^{3}$, Sz Burcsár ${ }^{3}$, M Kiszelák², B Vásárhelyi ${ }^{1}$ \\ ${ }^{1}$ Department of Laboratory Medicine, Faculty of Medicine, Semmelweis University, Budapest, Hungary \\ ${ }^{2}$ Diagon Ltd., Budapest, Hungary \\ ${ }^{3}$ Department of Rheumatology, Faculty of Medicine, Albert Szent-Györgyi Health Center, University of Szeged, \\ Szeged, Hungary
}

Received: September 8, 2016

Accepted: February 14, 2017

\begin{abstract}
Systemic lupus erythematosus (SLE) is a multisystemic inflammatory autoimmune disorder. Thrombotic events occur at a higher incidence among SLE patients. The investigation of thrombin generation (TG) with calibrated automated thrombogram (CAT) test as a global hemostasis assay is applicable for the overall functional assessment of the hemostasis. The aim of this study was to characterize the hemostatic alterations observed in SLE by CAT assay. In this study, CAT parameters and basic coagulation parameters of SLE patients $(n=22)$ and healthy control subjects $(n=34)$ were compared. CAT area under the curve (i.e., endogenous thrombin potential) was lower than normal in SLE (807 vs. $1,159 \mathrm{nM}^{*}$ min, respectively), whereas other CAT parameters (peak, lag time, time to peak, and velocity index) and the basic coagulation tests were within the normal range. The presence of anti-phospholipid antibodies and the applied therapy was not associated with hemostasis parameters in SLE. We concluded that the reported high risk of thrombosis is not related to TG potential.
\end{abstract}

Keywords: thrombin generation, systemic lupus erythematosus, thrombosis, endogenous thrombin potential, anti-phospholipid antibodies

\section{Introduction}

Systemic lupus erythematosus (SLE) is a multisystemic inflammatory autoimmune disorder that affects dominantly females. Thrombotic events occur with higher incidence and at a younger age among SLE patients compared with general population (2, 5); moreover, thrombosis is among the most common causes of death in SLE (4). In addition to the generally accepted risk factors, other predisposing factors are identified in SLE patients. These include the presence of anti-phospholipid (aPL) antibodies, ethnicity, older age, disease duration, disease activity, hypertension, nephritis, and corticosteroid treatment $(3,9)$. Among these factors the presence of aPL antibodies; particularly that of lupus anticoagulant (LAC) is associated with the highest risk of thrombosis $(18,20)$. In line with the high risk of thrombosis, elevated levels of prothrombin fragments were found at SLE patients with aPL antibodies (8) particularly at LAC-positive patients (7). The increased levels of prothrombin fragments demonstrate the prothrombotic state of these patients.

Thrombin generation (TG) tests are global hemostasis assays applicable for the overall functional assessment of the hemostasis (10). TG measurement is carried out by the detection of thrombin activity during the entire coagulation process provoked by the addition of small

Corresponding author: Anita Kuruczné Kern

Diagon Ltd., Baross utca 48-52, 1047 Budapest, Hungary

Phone: +36 1369 6500; Fax: +36 1369 3601; E-mail: akern@diagon.com 


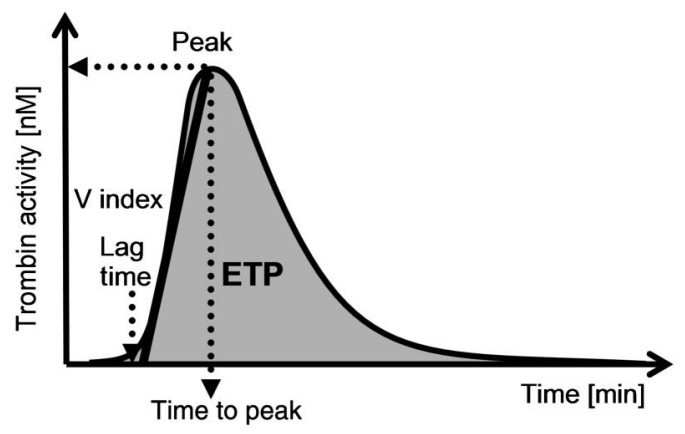

Fig. 1. Thrombogram. The graphical representation of the TG and its descriptive parameters. ETP: endogenous thrombin potential (area under the curve); $\mathrm{V}$ index: velocity index

amount of triggering agent to the sample. The monitored thrombin activity is graphically represented on the thrombogram and characterized by descriptive parameters of the TG curve (Fig. 1). Although TG measurements are still not standardized (11), commercially available tests are already in use to assess thrombotic risk in a number of disorders $(16,21)$.

Since TG can reliably reflect the general thrombotic or bleeding tendency (10), several studies applied TG assays for the exploration of the effects of different factors on hemostasis $(1,19)$ as well as for the investigation of the pathogenesis of the prothrombotic state in SLE $(6,13,14,17,19,22)$. These studies indicate that in the presence of aPL antibodies, the hypercoagulable state is characterized by activated protein $\mathrm{C}$ (aPC) resistance $(6,13,14,19,22)$ and they also suggest the involvement of microparticles (17). However, the results are inconsistent, probably, due to differences in the applied TG assays.

The aim of this study was to characterize the TG profile of SLE patients with calibrated automated thrombogram assay (CAT assay, a commercially available TG assay) and its association with clinical characteristics.

\section{Methods}

\section{Patients}

In total, 22 patients with various clinical characteristics of SLE and 34 non-affected genderand age-matched healthy control subjects were included in the study. Exclusion criteria were oral anticoagulant or heparin therapy. Clinical characteristics of patients are summarized in Table I. Systemic Lupus Erythematosus Disease Activity Index (SLEDAI) score was calculated for all patients at the time of sampling. Patients were treated with chloroquine $(n=13,59.1 \%)$, methylprednisolone $(n=18,81.8 \%)$ or prednisolone $(n=1,4.5 \%)$, azathioprine $(n=5,22.7 \%)$, cyclophosphamide $(n=4,18.2 \%)$, mycophenolate mofetil $(n=1$, $4.5 \%)$, epratuzumab $(n=1,4.5 \%)$, and methotrexate $(n=1,4.5 \%)$. Control subjects were age- and gender-matched healthy blood donors with no history of rheumatic symptoms recruited from the laboratory staff.

The project was approved by the Ethical Committee of the University of Szeged (ETTTUKEB 905/PI/09). Patients and healthy control subjects gave written informed consent to participate in the study. The study adhered to the tenets of Declaration of Helsinki.

\section{Laboratory measurements}

Venous blood samples were taken by venepuncture into Vacutainer ${ }^{\circledR}$ tubes (Becton Dickinson, Franklin Lakes, NJ, USA) containing 3.2\% sodium citrate. For standard coagulation 
Table I. Clinical characteristics of SLE patients and healthy control subjects. Qualitative data are expressed as number $(\%)$ and quantitative data as mean \pm standard deviation or median [1st-3rd interquartile], as appropriate

\begin{tabular}{|l|c|c|}
\hline & Patient & Control \\
\hline Number & 22 & 34 \\
\hline Age (years) & $48.9 \pm 11.2$ & $48.1 \pm 11.4$ \\
\hline Duration of SLE (years) & $3.5[0-14.5]$ & - \\
\hline SLEDAI & $6.0[3.5-8.5]$ & - \\
\hline CRP (mg/l) & $0[0-5.1]$ & $2.4[0-4.4]$ \\
\hline ESR (mm/h) & $22.0[8.0-40.8]$ & $9.8[4.0-14.0]$ \\
\hline C3 (mg/dl) & $90.4 \pm 39.0$ & n.d. \\
\hline C4 (mg/dl) & $14.2 \pm 6.9$ & n.d. \\
\hline aDNA (U/l) & $55.5[11.0-199.3]$ & n.d. \\
\hline aPL antibody positivity & $6(27.3 \%)$ & n.d. \\
\hline
\end{tabular}

aPL: anti-phospholipid antibodies; CRP: C-reactive protein; ESR: erythrocyte sedimentation rate; SLE: systemic lupus erythematosus; SLEDAI: Systemic Lupus Erythematosus Disease Activity Index; aDNA: anti-DNA antibody; n.d.: not determined

measurements, samples were centrifuged at 2,500 $\times g$ for $10 \mathrm{~min}$ at room temperature, and to obtain platelet poor plasma (PPP) for CAT measurements they were further centrifuged at $2,500 \times g$ for $20 \mathrm{~min}$. Samples were stored at $-80{ }^{\circ} \mathrm{C}$ until measurement (done within 1 month).

Standard coagulation measurements [activated partial thromboplastin time (aPTT), prothrombin time (PT), thrombin time (TT) and fibrinogen] were performed on Coag XL-automated coagulometer (Diagon Ltd., Budapest, Hungary) applying reagents from Diagon.

TG measurements were performed by applying CAT method (Diagnostica Stago, Gennevilliers, France). Briefly, $80 \mu$ platelet-free plasma was mixed with $20 \mu$ l trigger (PPP-Reagent, Thrombinoscope BV, Maastricht, The Netherlands) in round bottom 96-well microtiter plates (Immulon 2HB, Thermo Fisher Scientific Inc., Waltham, MA, USA). The measurement was started by the addition of $40 \mu \mathrm{l}$ FluCa-Kit (Thrombinoscope BV), which contained $\mathrm{CaCl}_{2}$ and fluorogenic thrombin substrate. Plasma samples were calibrated individually applying Thrombin Calibrator (Thrombinoscope BV). The generation of thrombin was measured with Fluoroskan Ascent reader (Thermo Fisher Scientific Inc.) at $390 \mathrm{~nm}$ excitation and $460 \mathrm{~nm}$ emission wavelengths. TG parameters [endogenous thrombin potential (ETP), peak, lag time, time to peak, and velocity index] were calculated by Thrombinoscope Software (Thrombinoscope BV). The measurements were done in duplicate.

\section{Statistical analysis}

Statistical analysis was performed by Minitab ${ }^{\circledR}$ Release 14.1 (Minitab Inc., State College, PA, USA). Normal distribution of data was examined by Anderson-Darling normality test. 
Table II. Comparison of standard coagulation tests and CAT parameters between SLE patients and control subjects. Data with normal distribution are expressed as mean \pm standard deviation and in case of non-normal distribution as median (1st-3rd interquartile)

\begin{tabular}{|l|c|c|c|}
\hline & Patient & Control & p \\
\hline PT (INR) & $1.04 \pm 0.09$ & $1.01 \pm 0.08$ & n.s. \\
\hline aPTT (s) & $32.0[29.5-36.0]$ & $36.2 \pm 3.3$ & $\mathbf{0 . 0 0 4 7}$ \\
\hline TT (s) & $19.2 \pm 1.6$ & $18.5[17.4-19.2]$ & n.s. \\
\hline Fibrinogen (g/l) & $3.04[2.71-3.28]$ & $2.88 \pm 0.47$ & n.s. \\
\hline ETP (nM*min) & $807 \pm 212$ & $1,159 \pm 262$ & $<\mathbf{0 . 0 0 0 5}$ \\
\hline Peak (nM) & $174 \pm 64$ & $198[151-258]$ & n.s. \\
\hline Lag time (min) & $3.03 \pm 0.82$ & $2.30[2.00-3.30]$ & n.s. \\
\hline Time to peak (min) & $5.77 \pm 1.24$ & $5.94 \pm 1.65$ & n.s. \\
\hline Velocity index (nM/min) & $61[38-100]$ & $58[36-109]$ & n.s. \\
\hline
\end{tabular}

Significant differences are marked with bold letters. aPTT: activated partial thromboplastin time; ETP: endogenous thrombin potential; INR: international normalized ratio; PT: prothrombin time; TT: thrombin time; n.s.: nonsignificant

Qualitative data were expressed as number (\%) and quantitative data as mean \pm standard deviation or median (1st-3rd interquartile), as appropriate. In case of normal distribution Student's $t$-test or in case of non-normal distribution Mann-Whitney $U$ test was applied to compare coagulation parameters between SLE patients and the control subjects, and between subgroups of patients. Patients with or without the following factors were compared as follows: aPL antibody positivity (27.3\%), anti-DNA antibody positivity $(59.1 \%)$, low C3 complement level (59.1\%), and low C4 complement level (22.7\%). Coagulation parameters of SLE patients were correlated with age, duration of SLE, SLEDAI, CRP, and ESR. Correlations were assessed with Pearson's or Spearman's rank coefficient, as appropriate. All tests were two-sided and $p$ values $<0.05$ were considered statistically significant.

\section{Results}

Standard coagulation tests and CAT parameters of 22 SLE patients and those of 34 control subjects were measured and compared (Table II). SLE patients had significantly shorter aPTT value than the control subjects; however, both values were within the reference range. Exclusion of patients with aPL antibodies did not affect the difference $(p=0.005)$. PT, TT, and fibrinogen values were not significantly different.

ETP value of SLE patients was significantly lower than that of control subjects, while other CAT parameters (peak, lag time, time to peak, and velocity index) were comparable. After the exclusion of patients with aPL antibodies, the difference between the ETP value of patients and control subjects remained significant $(p<0.0005)$, whereas other CAT parameters were not significantly different.

Comparison of SLE patient subgroups showed difference in the PT value of patients with and without aPL antibodies (INR 1.10 vs. INR 1.02, respectively, $p=0.0480$ ). Both 
values were within the normal range, and the difference was clinically irrelevant. No other difference in hemostasis tests including CAT parameters was observed between SLE patient subgroups formed in accordance with therapy or disease status.

None of the standard coagulation tests or any CAT parameters of SLE patients were associated with age, duration of SLE, SLEDAI, and CRP or applied therapy. Fibrinogen levels and TT values were correlated with ESR $(\rho=0.553, p=0.008$ and $\rho=0.508$, $p=0.016$, respectively) within SLE group.

\section{Discussion}

Epidemiologic studies have revealed several risk factors that may contribute to the higher risk of thrombotic events among SLE patients compared with general population. The underlying mechanism of the elevated thrombotic tendency in SLE is extensively investigated. These studies imply a complex mechanism characterized by aPC resistance, inhibition of TFPI, and involvement of microparticles.

TG test as a global hemostasis assay is expectedly an ideal approach for the characterization of complex hemostatic alterations. In this study, the TG profile of SLE patients was compared with that of control subjects. Significantly decreased ETP value was observed at SLE patients compared to control subjects, while other CAT parameters did not differ significantly. The basic coagulation test results were within the normal range for SLE patients and for control group.

Most studies report that for the detection of aPC resistance by TG measurement exogenous aPC need to be added to the sample $(6,13,14,19,22)$. However, a decreased ETP and prolonged lag time have been reportedly observed at LAC-positive patients (12), even in the absence of exogenous aPC. These results are in line with our findings of significantly reduced ETP (from 1,159 to $807 \mathrm{nM}^{*}$ min) and a not significant still remarkable (31.7\%) prolongation of lag time (from 2.30 to $3.03 \mathrm{~min}, p=0.0740$ ) in SLE. Since the aim of this study was to characterize the TG profile of SLE patients with a commercially available TG assay (namely CAT), no modification was made in the assay protocol provided by the manufacturer. However, by addition of aPC the CAT assay could have been used to determine the aPC resistance.

The most important risk factor for thrombosis in SLE is the presence of aPL antibodies. However, the association between aPL antibodies and TG parameters is controversial $(1,6,19,22)$, and it is suggested that sensitivity of TG alterations toward aPL antibodies are strongly affected by preanalytical conditions (15). Moreover, differences in the applied tests for the determination of aPL antibodies may result in controversial results. In this study, the effect of different aPL antibodies on coagulation test results was not compared due to the low number of aPL-positive patients (anti- $\beta 2$-glycoprotein alone, $n=3$; anti-cardiolipin alone, $n=1$; anti- $\beta 2$-glycoprotein and anti-cardiolipin, $n=2)$. In this study, no significant difference was observed in the CAT parameters of aPL-positive and aPL-negative SLE patients, which is in line with the findings of Boeer et al. (1) who applied a commercially available TG assay other than us. In these two studies, TG measurements were performed in accordance with the instructions of the manufacturers, and special preheating of the samples or addition of aPC were not applied. In this study, one patient with aPL antibodies had thrombophlebitis, whereas others did not show any thrombotic pattern. Moreover, the ratio of patients excluded from the study due to oral anticoagulant therapy following a previous 
thrombotic event were comparable between aPL-positive and aPL-negative SLE patients $(3 / 9=33 \%$ and $5 / 21=24 \%$, respectively $)$.

A limitation of the study is that only females were included in this study, however, SLE may also affect men. It is possible that hemostatic conditions of men differ from those of women, which should be further investigated.

In conclusion, we performed CAT measurements in SLE patients and found decreased ETP values. Of note, low ETP values are not consistent with the high thrombotic risk in SLE. In addition, ETP and other TG characteristics were not related to the presence of aPL antibodies and the applied therapy in SLE. This indicates that other factors than abnormal TG capacity contribute to the high thrombotic risk in SLE patients.

\section{Acknowledgement}

AB was supported by the János Bolyai Fellowship of the Hungarian Academy of Sciences.

\section{REFERENCES}

1. Boeer K, Cuznetov L, Loesche W: Thrombin generation as marker to estimate thrombosis risk in patients with abnormal test results in lupus anticoagulant routine diagnostics. Thromb. J. 11, 24 (2013)

2. Burgos PI, Alarcón GS: Thrombosis in systemic lupus erythematosus: risk and protection. Expert Rev. Cardiovasc. Ther. 7, 1541-1549 (2009)

3. Calvo-Alen J, Toloza SMA, Fernandez M, Barri HM, Fessler J, Roseman JM, McGwin G, Vila LM, Reveille JD, Alarcon GS, LUMINA Study Group: Systemic lupus erythematosus in a multiethnic US cohort (LUMINA), $\mathrm{XXV}$. Smoking, older age, disease activity, lupus anticoagulant, and glucocorticoid dose as risk factors for the occurrence of venous thrombosis in lupus patients. Arthritis Rheum. 52, 2060-2068 (2005)

4. Cervera R, Khamashta MA, Font J, Sebastiani GD, Gil A, Lavilla P, Mejía JC, Aydintug AO, ChwalinskaSadowska H, de Ramón E, Fernández-Nebro A, Galeazzi M, Valen M, Mathieu A, Houssiau F, Caro N, Alba P, Ramos-Casals M, Ingelmo M, Hughes GRV, European Working Party on Systemic Lupus Erythematosus: Morbidity and mortality in systemic lupus erythematosus during a 10-year period: a comparison of early and late manifestations in a cohort of 1,000 patients. Medicine (Baltimore) 82, 299-308 (2003)

5. Chung WS, Lin CL, Chang SN, Lu CC, Kao CH: Systemic lupus erythematosus increases the risks of deep vein thrombosis and pulmonary embolism: a nationwide cohort study. J. Thromb. Haemost. 12, 452-458 (2014)

6. Devreese K, Peerlinck K, Arnout J, Hoylaerts MF: Laboratory detection of the antiphospholipid syndrome via calibrated automated thrombography. Thromb. Haemost. 101, 185-196 (2009)

7. Ferro D, Quintarelli C, Valesini G, Violi F: Lupus anticoagulant and increased thrombin generation in patients with systemic lupus erythematosus [letter; comment]. Blood 83, 304 (1994)

8. Ginsberg JS, Demers C, Brill-Edwards P, Johnston M, Bona R, Burrows RF, Weitz J, Denburg JA: Increased thrombin generation and activity in patients with systemic lupus erythematosus and anticardiolipin antibodies: evidence for a prothrombotic state. Blood 81, 2958-2963 (1993)

9. Gustafsson JT, Gunnarsson I, Källberg H, Pettersson S, Zickert A, Vikerfors A, Möller S, Rönnelid J, Elvin K, Svenungsson E: Cigarette smoking, antiphospholipid antibodies and vascular events in systemic lupus erythematosus. Ann. Rheum. Dis. 74, 1537-1543 (2015)

10. Hemker HC, Al Dieri R, De Smedt E: Thrombin generation, a function test of the haemostatic-thrombotic system. J. Thromb. Haemost. 96, 553-561 (2006)

11. Kluft C, Meijer P: External quality assessment for thrombin generation tests: an exploration. Semin. Thromb. Hemost. 36, 791-796 (2010)

12. Liestøl S, Sandset PM, Jacobsen EM, Movinckel MC, Wisløff F: Decreased anticoagulant response to tissue factor pathway inhibitor type 1 plasmas from patients with lupus anticoagulants. Br. J. Haematol. 136, 131-137 (2006)

13. Liestøl S, Sandset PM, Mowinckel MC, Wisløff F: Activated protein C resistance determined with a thrombin generation-based test is associated with thrombotic events in patients with lupus anticoagulants. J. Thromb. Haemost. 5, 2204-2210 (2007) 
14. Meesters EW, Hansen H, Spronk HMH, Hamulyak K, Rosing J, Rowshani AT, ten Berge IJM, ten Cate H: The inflammation and coagulation cross-talk in patients with systemic lupus erythematosus. Blood Coagul. Fibrinolysis 18, 21-28 (2007)

15. Ninivaggi M, Klechtermans $\mathrm{H}$, Lindhout $\mathrm{T}$, de Laat B: Conformation of beta2glycoprotein I and its effect on coagulation. Thromb. Res. 130, S33-S36 (2012)

16. Panova-Noeva M, Marchetti M, Spronk HM, Russo L, Diani E, Finazzi G, Salmoiraghi S, Rambaldi A, Barbui T, Ten Cate H, Falanga A: Platelet-induced thrombin generation by the calibrated automated thrombogram assay is increased in patients with essential thrombocythemia and polycythemia vera. Am. J. Hematol. 86, 337-342 (2011)

17. Pereira J, Alfaro G, Goycoolea M, Quiroga T, Ocqueteau M, Massardo L, Pérez C, Sáez C, Panes O, Matus V, Mezzano D: Circulating platelet-derived microparticles in systemic lupus erythematosus. Association with increased thrombin generation and procoagulant state. Thromb. Haemost. 95, 94-99 (2006)

18. Petri M: Update on anti-phospholipid antibodies in SLE: the Hopkins' Lupus Cohort. Lupus 19, 419-423 (2010)

19. Regnault V, Béguin S, Wahl D, de Maistre E, Hemker HC, Lecompte T: Thrombinography shows acquired resistance to activated protein C in patients with lupus anticoagulants. Thromb. Haemost. 89, 208-212 (2003)

20. Somers E, Magder LS, Petri M: Antiphospholipid antibodies and incidence of venous thrombosis in a cohort of patients with systemic lupus erythematosus. J. Rheumatol. 12, 2531-2536 (2002)

21. Tripodi A, Legnani C, Chantarangkul V, Cosmi B, Palareti G, Mannucci PM: High thrombin generation measured in the presence of thrombomodulin is associated with an increased risk of recurrent venous thromboembolism. J. Thromb. Haemost. 6, 1327-1333 (2008)

22. Zuily S, Ait Aissa K, Membre A, Regnault V, Lecompte T, Wahl D: Thrombin generation in antiphospholipid syndrome. Lupus 21, 758-760 (2012) 